Part 1

\title{
Introductory reviews
}




\title{
New theoretical developments in stellar pulsation
}

\author{
Jørgen Christensen-Dalsgaard \\ Teoretisk Astrofysik Center, Danmarks Grundforskningsfond, and \\ Institut for Fysik og Astronomi, Aarhus Universitet, \\ DK-8000 Aarhus C, Denmark
}

\begin{abstract}
The basic properties of stellar oscillations are reasonably well understood, allowing measurements of their frequencies to be used as probes of stellar interiors. The detailed understanding of the processes responsible for the oscillations, in the very broad range of stars observed to pulsate, has improved substantially over the past decade, as have the techniques for asteroseismic investigations on the basis of the observations. Here I provide a brief overview of the theory of stellar oscillations, emphasizing several cases of recent progress, often inspired by new observational developments.
\end{abstract}

\section{Introduction}

Observations of stellar pulsation have made impressive progress in recent years. Large surveys have yielded extensive data on a variety of pulsating stars, in many cases for members of the local group of galaxies. Also, new techniques have allowed the observation of oscillations of very low amplitude, similar to those seen in the Sun. To use these data to obtain a better knowledge about stellar properties, we need to understand how the frequencies are related to those properties, and what are the causes of the pulsations.

Two distinct mechanisms are at work in the excitation of the oscillations. In one, the so-called $\kappa$-mechanism, phase relations between heating and compression in critical parts of the star work to cause overstability and hence intrinsic driving. This mechanism operates in the 'classical' pulsating stars in the instability strip but it has also been found to be active in a broad range of hotter stars, sometimes depending on enhancements of the critical chemical elements by radiative levitation. While the theoretical description of this mechanism generally accounts for the overall frequency range of unstable modes, the processes determining their limiting amplitudes, and hence their observability, are so far uncertain. In the second mechanism the modes are intrinsically stable but excited by stochastic driving by near-surface convection, resulting also in predictions of the mode amplitudes depending on the energy available in the convection. This was first identified as the source of the solar oscillations; their observed statistical properties and amplitudes are generally in rough agreement with theoretical predictions. Recently, solar-like oscillations have been identified in a broad range of stars, apparently extending also to semi-regular red-giant variables. In addition to opening exciting possibilities for asteroseismology of 
these stars, possibly also for stars in the Local Group, observations of such oscillations will yield valuable information about the properties of convection.

A complete discussion of the theory of stellar oscillations is obviously not possible here; for such discussions the book by Unno et al. (1989) or the review by Gough (1993) may be consulted, for example.

\section{Properties of oscillations}

I shall be concerned with general, so-called nonradial, oscillations, regarding as a special case the spherically symmetric, or radial, oscillations generally assumed for large-amplitude pulsators such as Cepheids. The variations of the oscillations with distance $r$ to the centre, co-latitude $\theta$ and longitude $\phi$, as well as time $t$, can then be expressed as, e.g.,

$$
V=\operatorname{Re}\left[v_{r}(r) Y_{l}^{m}(\theta, \phi) e^{-i \omega t}\right]
$$

for the radial component of velocity, where $Y_{l}^{m}(\theta, \phi)=c_{l m} P_{l}^{m}(\cos \theta) e^{i m \phi}$ is a spherical harmonic, $P_{l}^{m}$ being an associated Legendre function and $c_{l m}$ a normalization constant. Here the degree $l$ measures total horizontal wave number $k_{\mathrm{h}}=\sqrt{l(l+1)} / r$, and $m$ measures number of nodes in longitude; evidently radial modes correspond to $l=0$. The behaviour in the radial direction of the amplitude function $v_{r}(r)$ is characterized by the radial order $n$. In addition to the angular frequency $\omega$ we also use the cyclic frequency $\nu=\omega / 2 \pi=1 / \Pi$ where $\Pi$ is the period.

In general $\omega=\omega_{n l m}$ depends on $n, l$ and $m$. In particular, rotation induces a splitting of the frequencies according to $m$ which in principle allows inferences to be made about the internal rotation of stars; for moderate or rapid rotation the rotational effects substantially complicate the spectrum of oscillations. Here, however, I shall be concerned only with the effects of the spherically symmetric structure of the stars and hence I shall neglect the dependence on $m$ in the following. I note that, as a useful rough guide, the frequencies of a star scale approximately as the square root of the mean density, $\omega \propto\left(M / R^{3}\right)^{1 / 2}$.

\section{1. $\quad$ Mode properties}

The properties of the possible modes in a star are largely determined by two characteristic frequencies: the acoustic (or Lamb) frequency $S_{l}$, defined by

$$
S_{l}^{2}=\frac{l(l+1) c^{2}}{r^{2}}
$$

where $c$ is the adiabatic sound speed, and the buoyancy (or Brunt-Väisälä) frequency $N$, determined by

$$
N^{2}=g\left(\frac{1}{\Gamma_{1}} \frac{\mathrm{d} \ln p}{\mathrm{~d} r}-\frac{\mathrm{d} \ln \rho}{\mathrm{d} r}\right) \simeq \frac{g^{2} \rho}{p}\left(\nabla_{\mathrm{ad}}-\nabla+\nabla_{\mu}\right) .
$$

Here $g$ is the gravitational acceleration, $p$ is pressure, $\rho$ is density, and $\Gamma_{1}=$ $(\partial \ln p / \partial \ln \rho)_{\text {ad }}$; also, $c$ is given by $c^{2}=\Gamma_{1} p / \rho$. In Eq. (3) the second approximation assumes the ideal gas law, and $\nabla=\mathrm{d} \ln T / \mathrm{d} \ln p, \nabla_{\mathrm{ad}}=(\partial \ln T / \partial \ln p)_{\mathrm{ad}}$ and $\nabla_{\mu}=\mathrm{d} \ln \mu / \mathrm{d} \ln p$, where $T$ is temperature and $\mu$ is mean molecular weight. 
According to asymptotic theory the eigenfunctions oscillate as a function of $r$ where

$$
\omega^{2}>N^{2} \text { and } \omega^{2}>S_{l}^{2} \quad \text { (acoustic behaviour), }
$$

or

$$
\omega^{2}<N^{2} \text { and } \omega^{2}<S_{l}^{2} \quad \text { (gravity-wave behaviour). }
$$

Elsewhere the eigenfunction grows or decreases exponentially. Typically a mode is trapped, i.e., has substantial amplitude, only in one of the oscillatory regions, the eigenfunction decreasing exponentially outside it. If this region satisfies Eq. (4) the mode has the character of an acoustic, or p, mode, i.e., a standing acoustic wave. In the case of Eq. (5) the mode has the character of a gravity, or g, mode, i.e., a standing gravity wave. In 'normal' stars the p-mode behaviour is typically found in the outer part of the star, while g modes are trapped in the deep interior. ${ }^{1}$ As discussed in Section 4 cases of 'mixed' modes, with both pand g-mode character, are found in evolved stars.

An important quantity characterizing a mode of oscillation is its mode mass $M_{\text {mode }}$ or, equivalently, its inertia $E$, defined by

$$
M_{\text {mode }}=M E=\frac{\int_{V} \rho|\delta \mathbf{r}|^{2} \mathrm{~d} V}{\left|\boldsymbol{\delta} \mathbf{r}_{\mathrm{ph}}\right|^{2}},
$$

where $M$ is the mass of the star, $\delta \mathbf{r}$ is the displacement and $\delta \mathbf{r}_{\mathrm{ph}}$ is its photospheric value, and integration is over the volume $V$ of the star. With this definition, the total energy of oscillation is essentially given by $\mathcal{E}=M_{\text {mode }}\left|\mathbf{V}_{\mathrm{ph}}\right|^{2}$, where $\mathbf{V}_{\mathrm{ph}}$ is the photospheric velocity. Evidently, modes trapped in the deep interior of the star, typically g modes in stars other than white dwarfs, have a high mode mass and hence may crudely be expected to be more difficult to excite to observable amplitudes than modes trapped near the surface; this is particularly true of stochastically excited oscillators.

\subsection{Self-excitation or damping of stellar oscillations}

When excitation and damping is taken into account the mode depends on time as $\sim \cos (\omega t) e^{\eta t}$, with a growth rate $\eta$. The growth or decay of a mode is determined by the interaction between the energetics and the dynamics of the mode. Regions with net heating at compression act to destabilize the mode whereas regions that are cooled at compression act to stabilize it. The resulting growth rate, characterizing the combined effect of the different parts of the star, may be evaluated from

$$
\eta=-\frac{1}{2 \omega} \frac{\operatorname{Im}\left[\int_{V} \frac{\delta \rho^{*}}{\rho} \delta p \mathrm{~d} V\right]}{\int_{V} \rho|\boldsymbol{\delta} \mathbf{r}|^{2} \mathrm{~d} V}=\frac{1}{2 \omega^{2}} \frac{\operatorname{Re}\left[\int_{V} \frac{\delta \rho^{*}}{\rho}\left(\Gamma_{3}-1\right) \delta(\rho \epsilon-\operatorname{div} \mathbf{F}) \mathrm{d} V\right]}{\int_{V} \rho|\boldsymbol{\delta} \mathbf{r}|^{2} \mathrm{~d} V} .
$$

Here $\epsilon$ is the rate of energy generation, $\mathbf{F}$ is the energy flux and $\Gamma_{3}$ is defined by $\Gamma_{3}-1=(\partial \ln T / \partial \ln \rho)_{\text {ad }}$. The integration is over the volume $V$ of the star. Also, $\delta$ denotes the Lagrangian perturbation, i.e., the perturbation following the

\footnotetext{
${ }^{1}$ In white dwarfs, on the other hand, g modes are trapped in the outer parts of the star.
} 
motion. Note that the denominator in Eq. (7) is closely related to the mode mass (cf. Eq. 6).

The physical basis for instability was first outlined by Eddington (1926) and developed by Zhevakin (1953) and Cox \& Whitney (1958). If the opacity in a region is increased at compression, energy is dammed up and the region contributes to the driving. This typically occurs at an enhancement, or bump, in the opacity, such as in ionization zones of abundant elements. However, if this is to result in net instability, overcoming the damping in the rest of the star, the driving region must be located at the transition between nearly adiabatic and strongly nonadiabatic behaviour of the oscillation. Thus if the opacity bump is located at a radius $r_{\mathrm{b}}$, the thermal timescale of the layer outside $r_{\mathrm{b}}$ must be of order the pulsation period, i.e.,

$$
\Pi^{-1} L^{-1} \int_{r_{\mathrm{b}}}^{R} 4 \pi r^{2} \rho c_{p} T \mathrm{~d} r \sim 1
$$

where $L$ is the luminosity of the star and $c_{p}$ is the specific heat at constant pressure. This condition largely explains the location of the classical Cepheid instability strip, the relevant opacity bump being caused by the second ionization of helium. The location of a given opacity bump is typically determined by the temperature; thus with increasing effective temperature $T_{\text {eff }}$ the bump shifts closer to the surface. It follows from Eq. (8) that with increasing $T_{\text {eff }}$ the periods of the unstable modes tend to decrease.

Convection causes a major uncertainty in the calculation of the instability of the modes. To evaluate Eq. (7) we must determine the perturbations in the flux $\mathbf{F}$ and the pressure. This must include the contributions from the convective flux, which typically dominates energy transport in most of the convective regions, and turbulent pressure which provides a substantial contribution to hydrostatic balance very near the surface. Thus we need a procedure to evaluate convective properties in a time-dependent environment. Perhaps the most sophisticated such procedure was developed by Gough (1977) (see also Balmforth \& Gough 1990; Balmforth 1992) and applied to stability calculations by, for example, Baker \& Gough (1979) and Houdek (2000). The results indicate that stability of acoustic modes sets in on the cool side of the Cepheid instability strip as a result of the effects of the perturbations in the convective flux and turbulent pressure. However, convective effects are likely dominant in the excitation of long-period oscillations in the so-called $\gamma$ Doradus stars, on the cool side of the instability strip (Guzik et al. 2000; Warner et al. 2003). It was noted by Brickhill (1991), and later by Goldreich \& Wu (1999), that convective effects are also likely to dominate the instability of the cool pulsating DA white dwarfs (also known as ZZ Ceti stars).

Computations of stellar instability are reasonably successful in describing the occurrence of stellar pulsation in the HR diagram; some examples of this are discussed in the following. However, the theory as described so far provides no information about the expected amplitude of the resulting oscillation. In large-amplitude pulsators, such as Cepheids, the amplitude limitation appears to be caused by saturation of the driving mechanism. Closer to the main sequence, however, where amplitudes are typically smaller, it is likely that nonlinear interactions between unstable and stable modes are responsible for the 
limiting amplitudes and hence the observability of the modes (e.g., Dziembowski \& Królikowska 1985). However, the details are far from being understood.

\subsection{Stochastically excited oscillators}

In stars where all modes are intrinsically stable, oscillations may none the less be induced by forcing external to the oscillations. In single stars such forcing may result from the action of gas motions, most typically in vigorous near-surface convection in relatively cool stars, on the cool side of the Cepheid instability strip. This seems to be the source of the solar oscillations, and hence this type of oscillations is normally known as solar-like. In such a case, the amplitude of the oscillations is determined by the balance between the energy input from the forcing and the energy drain through the damping; thus, unlike the case of unstable modes, it is possible to make theoretical estimates of the expected amplitudes. It has been demonstrated that the statistical properties of the observed solar oscillation amplitudes are consistent with the assumption of stochastic excitation (Chaplin et al. 1997; Chang \& Gough 1998). Also, the energy input from convection was estimated from hydrodynamical convection simulations by Stein \& Nordlund (2001) and found to be consistent with the energy requirements determined from the observed amplitudes and damping rates.

The excitation by convection is dominated by the near-surface region, where the convective flows reach near-sonic velocities. As discussed, for example, by Goldreich et al. (1994) the frequency dependence of the amplitudes is determined by the energy spectrum of convection and the properties of the oscillation eigenfunctions. The most efficient driving results for modes with pulsation periods similar to the timescales of the convective eddies, i.e., in the solar case, periods of 5-10 min. Furthermore, at lower frequency the eigenfunctions are strongly reduced in the region of vigorous convection, compared with the amplitude in deeper layers, reducing the efficiency of the driving. At high frequency the energy input is reduced due to a lack of convective eddies with the corresponding timescales; also, strong damping sets in when the frequency is close to, or above, the acoustical cut-off frequency ( $\nu \simeq 5 \mathrm{mHz}$ in the solar case) where energy leakage through running waves in the atmosphere becomes possible. The result of these effects is a characteristic distribution of mode amplitudes, with a maximum for solar oscillations around a period of $5 \mathrm{~min}$ or a cyclic frequency of $3 \mathrm{mHz}$. It was argued by Brown et al. (1991) that the frequency of maximum amplitude should scale roughly as the acoustical cut-off frequency for other stars. The resulting frequencies, except in extreme red giants, correspond to acoustic modes of high radial order.

Estimates have been made of the amplitudes of oscillations in other stars, based on various models of the convective energy input and the damping processes. In a relatively simple analysis, using mixing-length theory of convection, Goldreich \& Keeley (1977) made an early estimate of the amplitude of solar oscillations, assuming that damping was also dominated by convective effects. They found that there is, very roughly, equipartition between the energy of one convective eddy at the timescale of the mode and one mode of oscillation:

$$
E_{\text {mode }} \sim M_{\text {mode }} V_{\mathrm{ph}}^{2} \sim E_{\text {eddy }}, \quad \text { or } \quad V_{\mathrm{ph}} \simeq f(\omega) M_{\text {mode }}^{-1 / 2}
$$


where the convective energy is assumed to depend only on the timescale, characterized by the oscillation frequency $\omega$, hence determining the function $f(\omega)$. Christensen-Dalsgaard \& Frandsen (1983) used this formulation to estimate amplitudes of stochastically excited radial oscillations in a broad range of stellar types. It was shown by Kjeldsen \& Bedding (1995) that the result could be approximated by a simple scaling law,

$$
\frac{V_{\mathrm{ph}}}{V_{\mathrm{ph}, \odot}} \sim \frac{L / L_{\odot}}{M / M_{\odot}}
$$

where $\odot$ refers to solar values. Houdek et al. (1999) used a more sophisticated non-local and time-dependent mixing-length description of convection, obtaining results that were similar to those of Christensen-Dalsgaard \& Frandsen. According to Eq. (9) the dependence of the amplitude on degree is contained in the dependence on $M_{\text {mode }}$, thus leading to lower amplitudes for modes that are partly trapped in the interior of the star. Additional reduction of the amplitudes may arise in cases where the damping of nonradial modes is dominated by the deep interior of the star, as discussed below.

\section{Overstable pulsators}

The overstable pulsators cover a broad range of stellar types. Here I concentrate on three groups of stars, with potential diagnostic value for studies of stellar properties. It might also be noted that the very interesting class of rapidly oscillating Ap stars, where magnetic fields play a crucial role for the pulsation properties, is discussed by Bigot (these proceedings).

\subsection{Double-mode pulsators}

In the instability strip some Cepheids, RR Lyrae stars and high-amplitude $\delta$ Scuti stars are observed to pulsate in two modes; these are generally assumed to be low-order radial modes, most often the fundamental and first overtone. The observed periods are in fact typically consistent with this assumption. Studies of the periods of double-mode pulsators may be considered as the first application of asteroseismology where observed oscillation periods were confronted with stellar models. It was noted by, for example, Petersen (1973) that the ratio $\Pi_{1} / \Pi_{0}$ between the fundamental and first overtone periods $\Pi_{0}$ and $\Pi_{1}$ provides information about stellar masses; this is often illustrated by means of the so-called Petersen $\left(\log \Pi_{0}, \Pi_{1} / \Pi_{0}\right)$ diagram. Early comparisons with stellar evolution models showed substantial discrepancies (e.g., Cox 1980). Simon (1982) and Andreasen \& Petersen (1988) pointed out that these could be alleviated by substantial changes in the opacities, and this has been dramatically confirmed by revised opacity calculations (e.g., Moskalik et al. 1992; Kanbur \& Simon 1994).

A remaining problem in the understanding of double-mode pulsators is the presence of a persistent two-mode behaviour. Linear stability calculations confirm that both modes are unstable in the relevant region of the HR diagram; however, initial attempts at non-linear analysis did not predict the simultaneous presence of the two modes, with stable amplitudes (for a review, see Kovács 1993). It has recently been demonstrated that the inclusion in the models of effects of turbulent convection may solve this problem. Feuchtinger (1998) 
found stable double-mode behaviour in non-linear hydrodynamical calculations of RR Lyrae stars, including convective effects. Similar results were obtained by Kolláth et al. (1998, 2002); in addition to non-linear calculations they carried out analyses of the oscillations in terms of truncated amplitude equations, elucidating more clearly the relevant properties of the oscillations. It is likely that further investigations along these lines, and similar investigations of other non-linear properties of pulsating stars (e.g., Feuchtinger 1999) may lead to new information about convection and its interaction with pulsations.

\subsection{Massive pulsating main-sequence stars}

Pulsations at hour-long periods are observed in the $\beta$ Cephei stars, which are main-sequence stars with masses in the range $10-15 M_{\odot}$. Also, at somewhat lower masses, long-period oscillations have been found in the so-called slowly pulsating B stars (SPB stars) (e.g., Waelkens 1991; Waelkens et al. 1998). The driving mechanism for these oscillations was for a long time a mystery; however, with the revised opacities instability was found for both $\beta$ Cephei and SPB stars, as a result of an opacity bump caused by iron-group elements (e.g., Dziembowski \& Pamyatnykh 1993; Dziembowski et al. 1993); the location of the region of instability is in striking agreement with observations (e.g., Waelkens et al. 1998). It should be noted that the presence of longer-period oscillators at lower effective temperature is in perfect accordance with the qualitative discussion of the conditions for instability above (cf. Eq. 8).

So far only a modest number of modes have been identified in most Bstar pulsators, limiting attempts at asteroseismic analysis of the frequencies (see, however, Dziembowski \& Jerzykiewicz 1996, 1999). Recently, Aerts et al. (2003a) presented first results of the analysis of a very long series of photometric data for the star HD 129929, determining frequencies of six modes; more detailed analysis of these data were presented by Aerts et al. (2003b) and Dupret et al. (2003). The results provided strict constraints on the structure of the star, including evidence for core overshoot, and indicated that the interior of the star rotates more rapidly than the surface. Similarly striking results on the $\beta$ Cephei star $\nu$ Eri are presented by Handler \& Aerts (these proceedings).

Given that the driving of the oscillations arises from opacity contributions from iron-group elements, instability may clearly be expected to depend on metallicity. This was confirmed by the detailed calculations by Pamyatnykh (1999); in particular, these indicated that it might be unlikely to find pulsating B stars in the Magellanic Clouds, given their comparatively low metallicity. Observationally, Kjeldsen \& Baade (1994) found weak evidence for $\beta$-Cephei-like variations in two stars in the SMC. Pigulski \& Kolaczkowski (2002) made a definite discovery of three $\beta$ Cephei stars in the LMC bar, from OGLE-II photometry. A major breakthrough in this field is reported by Kolaczkowski \& Pigulski (these proceedings), who have identified $64 \beta$ Cephei stars in the LMC, again based on OGLE-II data. A likely way to reconcile these observations with theory is to assume that iron has been locally enhanced in the region responsible for the driving, through radiative levitation and gravitational settling; such processes have been studied in detail in lower-mass stars (e.g., Turcotte et al. 1998, 2000). Similar calculations for more massive stars are urgently needed. It is evident that the results for the LMC pulsators may provide important observational 
constraints on these processes. (Note that the modelling of $\nu$ Eri discussed by Handler \& Aerts (these proceedings) also suggests that iron enhancement may be needed for instability in the model that best fits the frequency data.)

\subsection{Subdwarf B variables (sdBV stars)}

Pulsations amongst extreme blue horizontal-branch stars have been discovered in recent years. Interestingly, the pulsations were predicted theoretically by Charpinet et al. (1996) and discovered observationally by Kilkenny et al. (1997), independently and at essentially the same time. This class of pulsating stars is sometimes known as EC 14026 stars, from the initially-discovered member of the class. The stars have effective temperatures typically in excess of $30000 \mathrm{~K}$, with very thin hydrogen-rich envelopes, and are in the core helium burning phase of evolution. As for the $\beta$ Cephei stars the excitation is caused by the opacity mechanism operating on the opacity bump caused by iron-group elements; local enhancement of the iron abundance in the driving region appears to be required for instability (Charpinet et al. 1997). The stars typically show large numbers of excited modes and hence the observations have very substantial asteroseismic potential.

By analogy with the SPB stars, one might expect to find longer-period oscillations in horizontal-branch stars at slightly lower effective temperature. This was confirmed by observations made by Green et al. (2003) who found oscillations with periods of order one hour in stars with effective temperature between 25000 and $30000 \mathrm{~K}$. Fontaine et al. (2003) carried out a detailed theoretical analysis of these modes, confirming that they are likely $\mathrm{g}$ modes; it was found that radiative levitation of iron was again required to achieve instability. Interestingly, Fontaine et al. proposed that the observed modes have degree $l=3$ or 4 , since only for these values was instability found in the appropriate models, as a consequence of the detailed properties of the eigenfunctions. Such relatively high $l$-values are not a priori expected to dominate in stellar observations; however, even despite the substantial cancellation caused by the integration over the stellar disk the inferred physical amplitudes are probably not unreasonable. Further observations by other techniques are required to confirm this degree identification.

\section{Stochastically excited pulsators}

A major goal in the study of stellar pulsations has for a long time been the detection of solar-like oscillations in other stars; given the general nature of the excitation process, such oscillations would be expected in all stars with energetic near-surface convection although the predicted small amplitudes (below $1 \mathrm{~m} \mathrm{~s}^{-1}$ for main-sequence stars) evidently make detection very demanding. As reviewed by Bedding \& Kjeldsen (2003), the last few years mark a break-through in this field, largely as the result of the development of very stable techniques for radial-velocity observations. For bright stars observed with large telescopes the precision is approaching that achieved in observations of solar oscillations, e.g., making the oscillations directly visible in time series of observations of $\alpha$ Cen A with the UVES spectrograph on the VLT (Butler et al. 2003). 
As discussed above, solar-like oscillations are typically high-order acoustic modes. Their frequencies satisfy the asymptotic relation, to lowest order,

$$
\nu_{n l} \simeq \Delta \nu\left(n+\frac{l}{2}+\alpha\right)
$$

(Vandakurov 1967; Tassoul 1980), where $\Delta \nu=\left(2 \int_{0}^{R} \mathrm{~d} r / c\right)^{-1}$ is the inverse of the sound travel time across a stellar diameter and $\alpha$ is a surface phase. To this order Eq. (11) predicts that $\nu_{n-1 l+2} \simeq \nu_{n l}$; higher-order terms cause departures from this degeneracy. Thus the frequency spectrum is characterized by the large frequency separation $\Delta \nu_{n l}=\nu_{n l}-\nu_{n-1 l}$ and the small frequency separation $\delta \nu_{n l}=\nu_{n l}-\nu_{n-1 l+2} . \Delta \nu_{n l}$ provides information about the overall properties of the star; it is essentially proportional to $\left(M / R^{3}\right)^{1 / 2}$. On the other hand, $\delta \nu_{n l}$ is largely determined by the core properties and hence contains information about the extent to which hydrogen has been consumed in the star, reflecting its age. ${ }^{2}$ Together with the characteristic shape of the power spectrum resulting from stochastic excitation the frequency structure given by Eq. (11) is key to the observational identification of solar-like oscillations.

\subsection{Solar-like stars}

The initial analysis of observations of solar-like oscillations generally involves comparison with models of the large and, if available, the small frequency separations $\Delta \nu_{n l}$ and $\delta \nu_{n l}$. As discussed by, for example, Ulrich (1986) and Christensen-Dalsgaard (1988) these quantities in principle allow the determination of mass and age of main-sequence stars, although other uncertainties in the model parameters also need to be taken into account (Gough 1987, 2001). It is interesting that an initial analysis of this nature of data on $\alpha$ Cen $\mathrm{A}$ by Thévenin et al. (2002) indicated some discrepancies with the 'classical' data on the $\alpha$ Cen binary system.

With data of higher quality, allowing precise determination of individual frequencies, more detailed investigations of stellar internal properties are possible. An important aspect is the detection of effects of sharp features in stellar structure, varying on a scale smaller than the radial wavelength of the modes. Important examples are the properties at the base of the convective envelope (e.g., Monteiro et al. 2000) and the effect of the variation of sound speed associated with the second ionization of helium (e.g., Lopes et al. 1997; Pérez Hernández \& Christensen-Dalsgaard 1998). With sufficiently detailed data inverse analyses to infer the structure of the stellar core will become possible (e.g., Basu et al. 2002; Roxburgh 2002).

\subsection{Sub-giants}

According to Eq. (10) the amplitudes of solar-like oscillations are predicted to increase with stellar luminosity and hence larger amplitudes might be expected amongst sub-giants than on the main sequence. Indeed, the likely first identification of frequencies of solar-like oscillations was made by Kjeldsen et al. (1995)

\footnotetext{
${ }^{2}$ It was noted by Roxburgh \& Vorontsov (2003) that the ratio $\delta \nu_{n l} / \Delta \nu_{n l}$ provides a cleaner measure of the properties of the stellar core than does the small separation.
} 
in the sub-giant $\eta$ Boo, later confirmed in independent observations by Carrier et al. (2003). In such stars energy is generated in a hydrogen shell source outside a compact core. As a result the buoyancy frequency in the central parts of the star reaches substantial values, partly as a result of the high gravitational acceleration $g$, and partly in a region of steep hydrogen-abundance gradient just outside the core where $\nabla_{\mu}$ is large (cf. Eq. 3). As a result modes can behave like $\mathrm{g}$ modes in the deep interior at the high frequencies typical of stochastic excitation. This may give rise to mixed modes, with substantial amplitude both in a p-mode-like region in the outer parts of the star and a g-mode-like region in the core. For such modes the mode mass is not dramatically higher than for pure p modes and hence, according to Eq. (9), the modes may possibly be excited to observable amplitudes. However, the g-mode behaviour in the core causes departures in the frequencies from the pure p-mode asymptotic behaviour in Eq. (11). These properties are evident in computed frequencies for models of $\eta$ Boo (Christensen-Dalsgaard et al. 1995; Guenther \& Demarque 1996; Di Mauro et al. 2003). Interestingly, the observed frequencies may show hints of a similar behaviour; if this is confirmed by more extensive observations it would provide a possibility for detailed study of the stratification of the deep interior of such stars.

\subsection{Late $\mathrm{G}, \mathrm{K}$ giants}

For even more evolved stars larger amplitudes are expected. Frandsen et al. (2002) analyzed an extended series of observations of the G7 giant $\xi$ Hydrae and found clear evidence for solar-like oscillations. In accordance with the much lower acoustical cut-off frequency of this star the maximum in power is around a period of $3 \mathrm{hr}$, but the power distribution in the spectrum is otherwise quite similar to that observed in the Sun and other main-sequence stars, with an apparent series of evenly spaced peaks in superficial agreement with Eq. (11). From its location in the HR diagram it is likely that the star is in the core helium burning phase, with a highly condensed core and consequently very high values of the buoyancy frequency in the deep interior. For $l>0$ this gives rise to an extremely dense spectrum of g modes with substantial amplitudes in the core, interspersed with modes that are predominantly of acoustic nature. It was noted by Dziembowski et al. (2001) that even for these largely acoustic modes the very rapid variation of the eigenfunction in the g-mode region in the core is likely to lead to substantial radiative damping and hence a reduced amplitude, compared with the radial modes where this effect is obviously absent. Accordingly, the observed spectrum should probably be interpreted as dominated by the radial modes, with the spacing between the peaks given by $\Delta \nu$, rather than the spacing of $\Delta \nu / 2$ which in accordance with Eq. (11) is observed in main-sequency stars between modes of alternating even and odd degree. In fact, the observed frequency spacing is consistent with this interpretation, given the stellar properties inferred from its effective temperature and luminosity.

\subsection{Red-giant semiregular variables}

Moving up the red-giant branch a further increase in the amplitudes of stochastically excited oscillations would be expected. Indeed, as discussed by Kiss \& Bedding (these proceedings; see also Kiss \& Bedding 2003) OGLE-II data on 
LMC stars indicate that variability is ubiquitous amongst sufficiently bright red giants, the lower limit in the survey being set by observational limitations. At lower luminosity Edmonds \& Gilliland (1996) found low-amplitude variability in $\mathrm{K}$ giants while oscillations have also been found in Arcturus (e.g., Retter et al. 2003). At the other extreme, large-amplitude variability is found in the Mira and semi-regular variables. An observational problem for these variables are their extremely long periods, of order months or years; however, as a result of their large amplitudes very valuable data have been obtained from amateur observations, stretching over many decades.

Mattei et al. (1997) analyzed a large set of data from the American Association of Variable Star Observers (AAVSO) in terms of amplitude and variability of the amplitude. They showed that the variables fall in two distinct classes: the Miras with large amplitude and modest amplitude variability, and the semiregular variables with comparatively low amplitudes and high variability; for the latter class there appeared to be a roughly linear relation between amplitude and its variability. It was pointed out by Christensen-Dalsgaard et al. (2001) that this behaviour would indeed be expected from the exponential energy distribution in stochastically excited oscillators (e.g., Kumar et al. 1988; Chang \& Gough 1998); for this distribution the standard deviation $\sigma(A)$ of the amplitude is related to the average amplitude $\langle A\rangle$ by $\sigma(A)=(4 / \pi-1)^{1 / 2}\langle A\rangle \simeq 0.52\langle A\rangle$, approximately consistent with the observed distribution. This suggests that the physics of stochastic excitation, and the properties of convection, can be studied over an extremely broad range of stellar parameters from observations of solar-like oscillations.

Acknowledgments. This work was supported in part by the Danish National Research Foundation through its establishment of the Theoretical Astrophysics Center.

\section{References}

Aerts, C., Thoul, A., Daszyńska, J., Scuflaire, R., Waelkens, C., Dupret, M.A., Niemczura, E., Noels, A. 2003a, Science, 300, 1926

Aerts, C., Waelkens, C., Daszyńska-Daszkiewicz, J., Dupret, M.-A., Thoul, A., Scuflaire, R., Uytterhoeven, K., Niemczura, E., Noels, A. 2003b, A\&A, in press

Andreasen, G.K., Petersen, J.O. 1988, A\&A, 192, L4

Baker, N.H., Gough, D.O. 1979, ApJ, 234, 232

Balmforth, N.J. 1992, MNRAS, 255, 603

Balmforth, N.J., Gough, D.O. 1990, Solar Phys., 128, 161

Basu, S., Christensen-Dalsgaard, J., Thompson, M.J. 2002, in Proc. 1st Eddington Workshop, 'Stellar Structure and Habitable Planet Finding', eds F. Favata, I.W. Roxburgh \& D. Galadí-Enríquez, ESA SP-485 (Noordwijk: ESA), 249

Bedding, T.R., Kjeldsen, H. 2003, Publ. Astron. Soc. Australia, 20, 203

Brickhill, A.J. 1991, MNRAS, 251, 673

Brown, T.M., Gilliland, R.L., Noyes, R.W., Ramsey, L.W. 1991, ApJ, 368, 599 
Butler, R.P., Bedding, T.R., Kjeldsen, H., McCarthy, C., O'Toole, S.J., Tinney, C.G., Marcy, G.W., Wright, J.T. 2003, ApJL, in press

Carrier, F., Bouchy, F., Eggenberger, P. 2003, in Asteroseismology across the HR diagram, eds M.J. Thompson, M.S. Cunha \& M.J.P.F.G. Monteiro (Dordrecht: Kluwer), 315

Chang, H.-Y., Gough, D.O. 1998, Solar Phys., 181, 251

Chaplin, W.J., Elsworth, Y., Howe, R., Isaak, G.R., McLeod, C.P., Miller, B. A., New, R. 1997, MNRAS, 287, 51

Charpinet, S., Fontaine, G., Brassard, P., Dorman, B. 1996, ApJ, 471, L103

Charpinet, S., Fontaine, G., Brassard, P., Chayer, P., Rogers, F., Iglesias, C.A, Dorman, B. 1997, ApJ, 483, L123

Christensen-Dalsgaard, J. 1988, in IAU Symp. 123, Advances in helio- and asteroseismology, eds J. Christensen-Dalsgaard \& S. Frandsen, (Dordrecht: Reidel), 295

Christensen-Dalsgaard, J., Frandsen, S. 1983, Solar Phys., 82, 469

Christensen-Dalsgaard, J., Bedding, T.R., Kjeldsen, H. 1995, ApJ, 443, L29

Christensen-Dalsgaard, J., Kjeldsen, H., Mattei, J.A. 2001, ApJ, 562, L141

Cox, A.N. 1980, ARA\&A, 18, 15

Cox, J.P., Whitney, C. 1958, ApJ, 127, 561

Di Mauro, M.P., Christensen-Dalsgaard, J., Kjeldsen, H., Bedding, T.R., Paternò, L. 2003, A\&A, 404, 341

Dupret, M.-A., Thoul, A., Scuflaire, R., Daszyńska-Daszkiewicz, J., Aerts, C., Bourge, P.-O., Waelkens, C., Noels, A. 2003, A\&A, in press

Dziembowski, W.A., Jerzykiewicz, M. 1996, A\&A, 306, 436

Dziembowski, W.A., Jerzykiewicz, M. 1999, A\&A, 341, 480

Dziembowski, W., Królikowska, M. 1985, Acta Astron., 35, 5

Dziembowski, W.A., Pamyatnykh, A.A. 1993, MNRAS, 262, 204

Dziembowski, W.A., Gough, D.O., Houdek, G., Sienkiewicz, R. 2001, MNRAS, 328,601

Dziembowski, W.A., Moskalik, P., Pamyatnykh, A.A. 1993, MNRAS, 265, 588

Eddington, A.S. 1926, The internal constitution of the stars, (Cambridge: Cambridge University Press)

Edmonds, P.D., Gilliland, R.L. 1996, ApJ, 464, L157

Feuchtinger, M.U. 1998, A\&A, 337, L29

Feuchtinger, M.U. 1999, A\&A, 351, 103

Fontaine, G., Brassard, P., Charpinet, S., Green, E.M., Chayer, P., Billères, M., Randall, S.K. 2003, ApJ, 597, 518

Frandsen, S., Carrier, F., Aerts, C., Stello, D., Maas, T., Burnet, M., Bruntt, H., Teixeira, T.C., de Medeiros, J.R., Bouchy, F., Kjeldsen, H., Pijpers, F., Christensen-Dalsgaard, J. 2002, A\&A, 394, L5

Goldreich, P., Keeley, D.A. 1977, ApJ, 212, 243

Goldreich, P., Wu, Y. 1999, ApJ, 511, 904

Goldreich, P., Murray, N., Kumar, P. 1994, ApJ, 424, 466 
Gough, D.O. 1977, ApJ, 214, 196

Gough, D.O. 1987, Nature, 326, 257

Gough, D.O. 1993, in Les Houches Session XLVII, Astrophysical fluid dynamics, eds J.-P. Zahn \& J. Zinn-Justin (Amsterdam: Elsevier), 399

Gough, D.O. 2001, in ASP Conf. Ser. Vol. 245, Astrophysical Ages and Time Scales, eds T. von Hippel, C. Simpson \& N. Manset (San Francisco: ASP), 31

Green, E.M., Fontaine, G., Reed, M.D., Callerame, K., Seitenzahl, I.R., White, B.A., Hyde, E.A., Østesen, R., Cordes, O., Brassard, P., Falter, S., Jeffery, E.J., Dreizler, S., Schuh, S.L., Giovanni, M., Edelmann, H., Rigby, J., Bronowska, A. 2003, ApJ, 583, L31

Guenther, D.B., Demarque, P. 1996, ApJ, 456, 798

Guzik, J.A., Kaye, A.B., Bradley, P.A., Cox, A.N., Neuforge, C. 2000, ApJ, 542, L57

Houdek, G. 2000, in ASP Conf. Ser. Vol. 210, Delta Scuti and related stars, eds M. Breger \& M.H. Montgomery (San Francisco: ASP), 454

Houdek, G., Balmforth, N.J., Christensen-Dalsgaard, J., Gough, D.O. 1999, A\&A, 351, 582

Kanbur, S.M., Simon, N.R. 1994, ApJ, 420, 880

Kilkenny, D., Koen, C., O'Donoghue, D., Stobie, R.S. 1997, MNRAS, 285, 640

Kiss, L.L., Bedding, T.R. 2003, MNRAS, 343, L79

Kjeldsen, H., Baade, D. 1994, in IAU Symp. 162, Pulsation, rotation and mass loss in early-type stars, eds L.A. Balona, H.F. Henrichs \& J.M. Contel (Dordrecht: Kluwer), 29

Kjeldsen, H., Bedding, T.R. 1995, A\&A, 293, 87

Kjeldsen, H., Bedding, T.R., Viskum, M., Frandsen, S. 1995, A\&A, 109, 1313

Kolláth, Z., Beaulieu, J.P., Buchler, J.R., Yecko, P. 1998, ApJ, 502, L55

Kolláth, Z., Buchler, J.R., Szabó, R., Csubry, Z. 2002, A\&A, 385, 932

Kovács, G. 1993, Ap\&SS, 210, 281

Kumar, P., Franklin, J., Goldreich, P. 1988, ApJ, 328, 879

Lopes, I., Turck-Chièze, S., Michel, E., Goupil, M.-J. 1997, ApJ, 480, 794

Mattei, J.A., Foster, G., Hurwitz, L.A., Malatesta, K.H., Willson, L.A., Mennissier, M.-O. 1997, in Proc. ESA Symposium: Hipparcos - Venice '97, eds B. Battrick, M.A.C. Perryman, P.L. Bernacca \& K.S. O'Flaherty, ESA SP-402 (Noordwijk: ESA), 269

Monteiro, M.J.P.F.G., Christensen-Dalsgaard, J., Thompson, M.J. 2000, MNRAS, 316, 165

Moskalik, P., Buchler, J.R., Marom, A. 1992, ApJ, 385, 685

Pérez Hernández, F., Christensen-Dalsgaard, J. 1998, MNRAS, 295, 344

Pamyatnykh, A.A. 1999, Acta Astron., 49, 119

Petersen, J.O. 1973, A\&A, 27, 89

Pigulski, A., Kolaczkowski, Z. 2002, A\&A, 388, 88 
Retter, A., Bedding, T.R., Buzasi, D.L., Kjeldsen, H., Kiss, L.L. 2003, ApJ, 591, L151 (Erratum: ApJ, 596, L125)

Roxburgh, I.W. 2002, in Proc. 1st Eddington Workshop: Stellar structure and habitable planet finding, eds F. Favata, I.W. Roxburgh \& D. GaladíEnríquez, ESA SP-485 (Noordwijk: ESA), 75

Roxburgh, I.W., Vorontsov, S.V. 2003, A\&A, 411, 215

Simon, N.R. 1982, ApJ, 260, L87

Stein, R.F., Nordlund, $\AA .2001$, ApJ, 546, 585

Tassoul, M. 1980, ApJS, 43, 469

Thévenin, F., Provost, J., Morel, P., Berthomieu, G., Bouchy, F., Carrier, F. 2002, A\&A, 392, L9

Turcotte, S., Richer, J., Michaud, G. 1998, ApJ, 504, 559

Turcotte, S., Richer, J., Michaud, G., Christensen-Dalsgaard, J. 2000, A\&A, 360,603

Ulrich, R.K. 1986, ApJ, 306, L37

Unno, W., Osaki, Y., Ando, H., Saio, H., Shibahashi, H. 1989, Nonradial Oscillations of Stars, 2nd Edition (Tokyo: University of Tokyo Press)

Vandakurov, Yu.V. 1967, AZh, 44, 786 (English translation: Soviet Ast., 11, 630)

Waelkens, C. 1991, A\&A, 246, 453

Waelkens, C., Aerts, C., Kestens, E., Grenon, M., Eyer, L. 1998, A\&A, 330, 215

Warner, P.B., Kaye, A.B., Guzik, J.A. 2003, ApJ, 593, 1049

Zhevakin, S.A. 1953, AZh, 30, 161

\section{Discussion}

Kawaler: The He burning core in a star should be convective, but your figure didn't clearly show that in the Brunt-Väisälä frequency. Can you describe the core structure in terms of the Brunt-Väisälä frequency?

Christensen-Dalsgaard: The model does indeed have a convective core but with a radius of only $0.002 \mathrm{R}$; thus it was not visible on the plot including the entire model.

Percy: You showed the power spectrum for a MACHO pulsating red giant. How can you tell, from the observations, whether the pulsations are stochastically excited?

Christensen-Dalsgaard: The spectrum shows peaks with roughly Lorentzian shape, but fine structure under the Lorentzian envelopes as seen also for solar oscillations. A more definite demonstration of the stochastic nature would require analysis of the statistics of the mode amplitudes, as functions of time. 


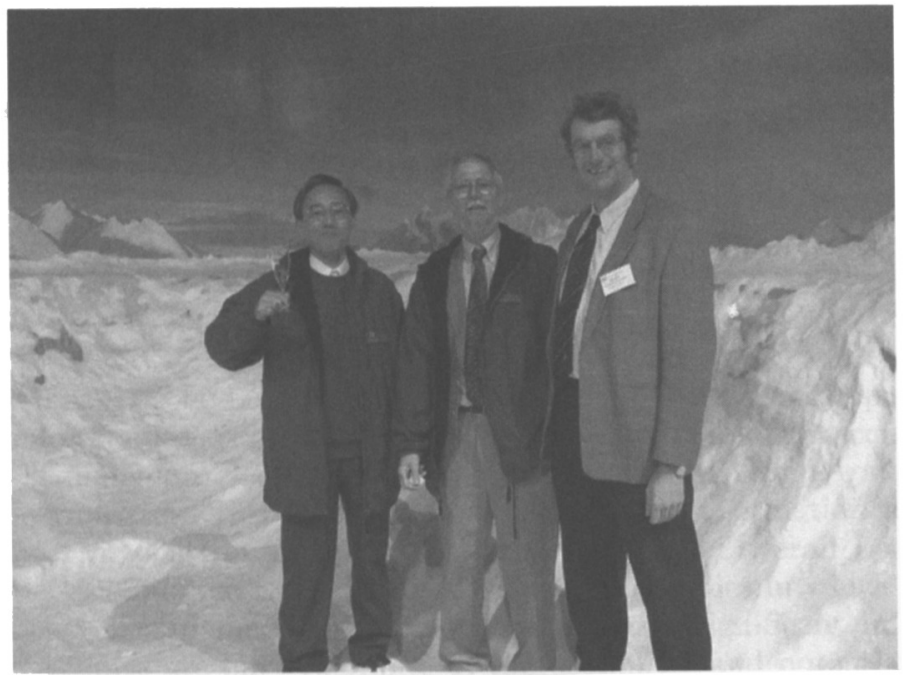

Hiromoto Shibahashi, Don Kurtz and Jørgen Christensen-Dalsgaard

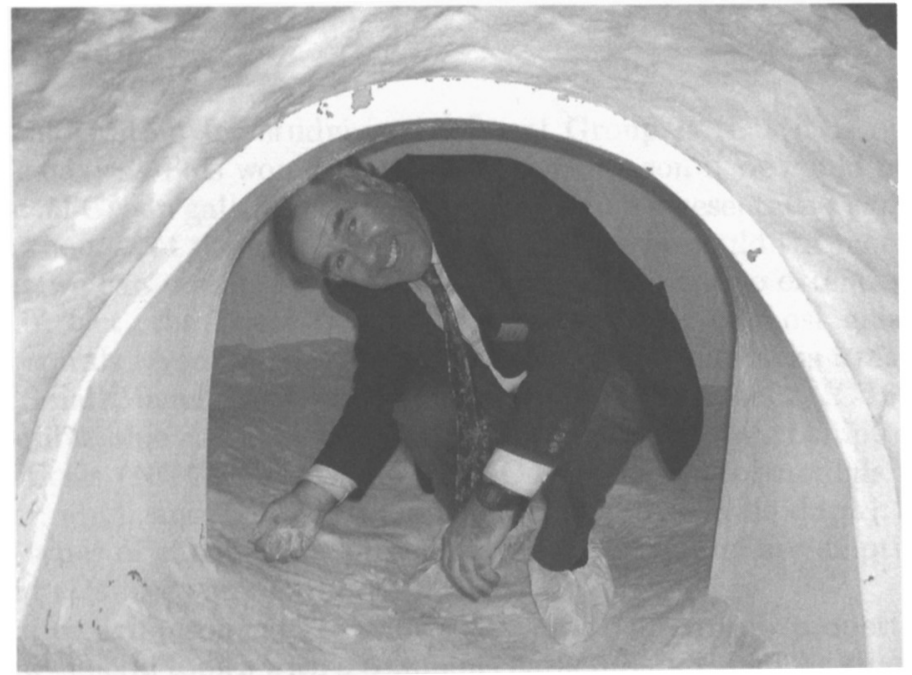

John Hearnshaw 